\title{
Characterization of X-ray emission from laser generated plasma
}

\author{
Antonino Cannavò ${ }^{1, *}$, Lorenzo Torrisi $^{1}$, Giovanni Ceccio $^{1}$, Mariapompea Cutroneo $^{2}$, Lucia $^{5}$ Calcagno $^{3}$, Antonella Sciuto $^{4}$ \\ and Massimo Mazzillo ${ }^{5}$ \\ ${ }^{1}$ Dottorato di Ricerca \& Dip.to di Scienze Fisiche MIFT, Università di Messina, V.F.S. D’Alcontres 31, 98166 S. Agata(ME), Italy \\ ${ }^{2}$ Physics Institute, ASCR, 25068 Rez, Czech Republic \\ ${ }^{3}$ Dipartimento di Fisica \& Astronomia, Università di Catania, V. S. Sofia 64, 95123 Catania, Italy \\ ${ }^{4}$ CNR-IMM, VIII strada $\mathrm{n}, 95121$ Catania, Italy \\ ${ }^{5}$ ST-Microelectronics, Str.le Primosole 50, 95121 Catania, Italy
}

\begin{abstract}
X-ray emission from laser generated plasma was studied at low $\left(10^{10} \mathrm{~W} / \mathrm{cm}^{2}\right)$ and high $\left(10^{18}\right.$ $\mathrm{W} / \mathrm{cm}^{2}$ ) intensity using $\mathrm{ns}$ and fs laser, respectively. Plasma characteristics were controlled trough the laser parameters, the irradiation conditions and the target properties. The X-ray spectra were acquired using fast detection technique based on $\mathrm{SiC}$ diodes with different active regions. The X-ray yield increases with the atomic number of the target, both at low and high intensity, and a similar empirical law has been obtained. The X-ray emission mechanisms from plasma are correlated to the plasma temperature and density and to the Coulomb charge particle acceleration, due to the charge separation effects produced in the non-equilibrium plasma. Functional dependences, theoretical approaches and interpretation of possible mechanism will be presented and discussed.
\end{abstract}

\section{Introduction}

When a high intensity laser pulse $\left(I=10^{10}-10^{18} \mathrm{~W} / \mathrm{cm}^{2}\right)$ is focused on the surface of a target placed in vacuum the generated plasma acts as source of photons, from IR to Xrays regions, high energetic and thermal electrons and ions. So the possibility to obtain very compact X-rays source with high time and space resolution has given a great impulse in this research field.

From the theoretical point of view the laser-matter interaction and the consequent $\mathrm{X}$-rays production has been widely reported in literature [1]. There are three fundamental emission processes due to free-free, freebound and bound-bound electrons transitions. In the first case, the emission in a continuum spectrum is due to the interaction between electrons and the Coulomb potential of ions, the so called bremsstrahlung effect. The second process leads again to the emission of a continuum spectrum and is due to the re-combination phenomena through the passage between an initial free electron state to a bound electron state. The last mechanism produces line spectrum due to transition between discrete energy levels [2]. The ratio in which Bremmstrahlung or recombination phenomena occurs, is strongly influenced by the atomic number of the target. In particular, the ratio of the spectrally integrated recombination $\left(W_{r}\right)$ and Bremsstrahlung $\left(W_{B}\right)$ intensities:

$$
\frac{W r}{W_{B}}=2.4 \frac{Z^{2}}{E_{H}} k_{B} T
$$

in which $E_{H}=13.6 \mathrm{eV}$ is the Hydrogen ionization energy, $\mathrm{Z}$ is the atomic number and $k_{B} T$ is the plasma temperature. For low $\mathrm{Z}$ and/or high temperature plasmas, the Bremsstrahlung emission overcomes the recombination emission. Concerning the X-rays pulse duration, the overall emission is comparable with the laser pulse but changes according to the considered spectral range: the higher is the energy of the considered photons the shorter is the duration of the plasma emission. Finally the peak of $\mathrm{X}$-ray emissivity is located in the plasma region which combines both high temperature and high electron density, typically just beyond the critical surface density.

\section{Experimental set-up}

The experiments were carried out using the so called time of flight technique (TOF) in which the radiations energy can be determined by the time to cross a known distance. The electronic chain is reported in Fig. 1a and consists in a fast storage oscilloscope (Tektronix TDS5104B, 5 Gs/s sampling rate) connected to the detector through a coupling capacitance $\mathrm{C}$ while the detector is biased independently via the resistance $\mathrm{R}$.

Choosing the appropriate values for $\mathrm{R}$ and $\mathrm{C}$, which determine the decay constant of the electronics, it is possible to put in evidence the very fast component of the spectrum, essentially due to the prompt photon and electron emissions, or to the slow component due to ions detection. There are many advantages in the use of the TOF technique. First of all, it gives the possibility to

Corresponding author: acannavo@unime.it 


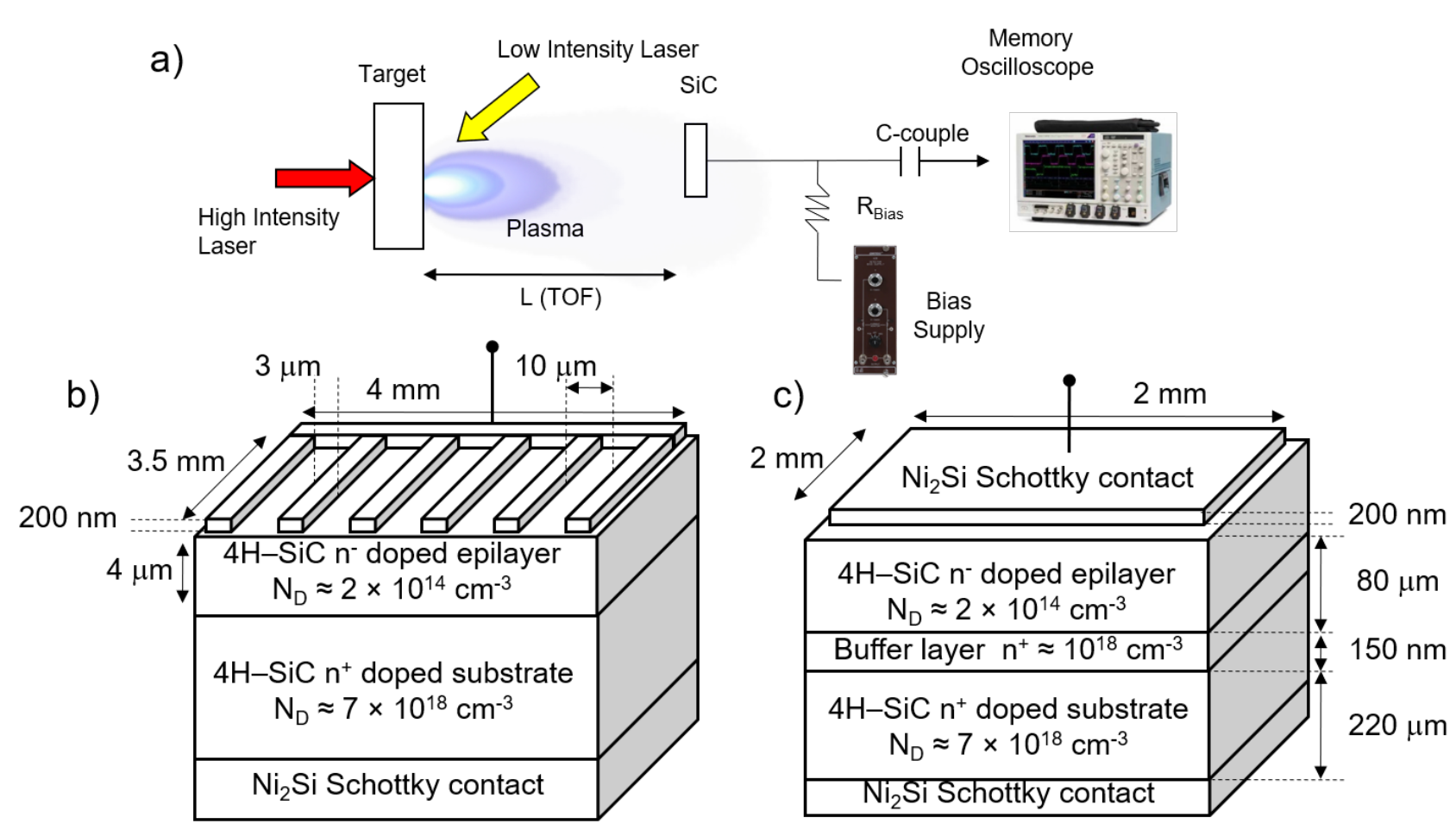

Fig. 1. Description of the TOF technique set-up a) and of the geometry of the SiC AZ 80 b) and SiC IAZ4 c) detector.

monitor very well high particle flux, without any problems concerning pile-up effects. This technique gives also time information because it is based on the time of arrival of radiation to the detector.

Finally it can be applied in a wide range of laser intensities and conditions. In fact the results presented in this work have been acquired using three lasers operating in Italy, in Czech Republic and in France:

- Nd:YAG laser, 1064 nm, 3 ns, $10^{10} \mathrm{~W} / \mathrm{cm}^{2} @$ UniME; - Iodine laser, 1315 nm, 300 ps, 10 16 W/cm²@PALS; - Ti Sapphire laser, 800 nm, 10 $0^{18}$ W/cm2@, CELIA.

The disadvantage to use TOF approach for photon measurements consists in the photon energy evaluation which is not immediate. In fact, while the energy of the particles can be determined with high precision from their time of flight from the target to the detector, photons arrive almost instantaneously to the detector, travelling with a velocity $\mathrm{c}$ along limited flight distances of the order of $1 \mathrm{~m}$. However, some estimation about the mean energy of photon can be done by using different absorbers placed in front of the detector, as described in [3]. The used detectors are based on Silicon Carbide (SiC) material. Thanks to the physical properties of this semiconductor, the detectors have a high temperature operability (up to $\left.1240{ }^{\circ} \mathrm{C}\right)$, fast response $\left(900 \mathrm{~cm}^{2} / \mathrm{Vs}\right.$ electron mobility and $110 \mathrm{~cm}^{2} / \mathrm{Vs}$ holes mobility), and they can be used with high electrical field having a breakdown electric field of $3 \mathrm{MV} / \mathrm{cm}$. Moreover they have got peculiar characteristics to be employed in harsh environment such that which characterize the diagnostic of plasma generated by laser. $\mathrm{SiC}$ detectors have an high resistance to damage due to radiations thanks to the higher displacement energy (25 $\mathrm{eV})$ with respect to Silicon $(13.5 \mathrm{eV})$. But the key point is the fact that because of the high value of the bandgap these detectors cut the very high intensity visible radiation emitted from the laser-generated plasmas, which represents the main source of noise.

The used devices, schematized in Fig. $1 \mathrm{~b}$ and 1c, were designed and fabricated in the STMicroelectronics and CNR-IMM clean room facilities and are widely described in previous paper [4].

They have different geometry of the top metallization layer and different thickness of the n-doped $4 \mathrm{H}-\mathrm{SiC}$ sensible layer.

They give complementary information being the first device, labeled SiC IAZ4, able to detect low energy ions, electrons, soft X-rays and UV [5] due to the active region directly exposed to radiation and to the low thickness of the active region $(4 \mu \mathrm{m})$. On the other hand the second detector, labeled $\mathrm{SiC} \mathrm{AZ}$ 80, presents a thick top metallization $\left(\mathrm{Ni}_{2} \mathrm{Si}, 200 \mathrm{~nm}\right.$ thick) and a deep active region about $80 \mu \mathrm{m}$ applying $600 \mathrm{~V}$ and for these reasons is very efficient in detecting hard X-rays and high ions energy [6].

\section{Results}

A typical TOF spectrum, acquired at PALS facility, is shown in Fig. 2.

It was obtained irradiating a polyethylene target $(10 \mu \mathrm{m}$ thick) and detecting through the $\mathrm{SiC} \mathrm{AZ} 80$ placed in forward direction, at $0^{\circ}$ with a flight distance of $72 \mathrm{~cm}$. It presents a fast intense peak, called photopeak, due to hard X-rays and UV.

It is followed by an edge due to hot relativistic electrons and a little tail due to cold electrons.

Instead the last wide structure is due to protons, to carbon ions and at time of flight higher than $150 \mathrm{~ns}$ to the massive slower CxHy groups. 


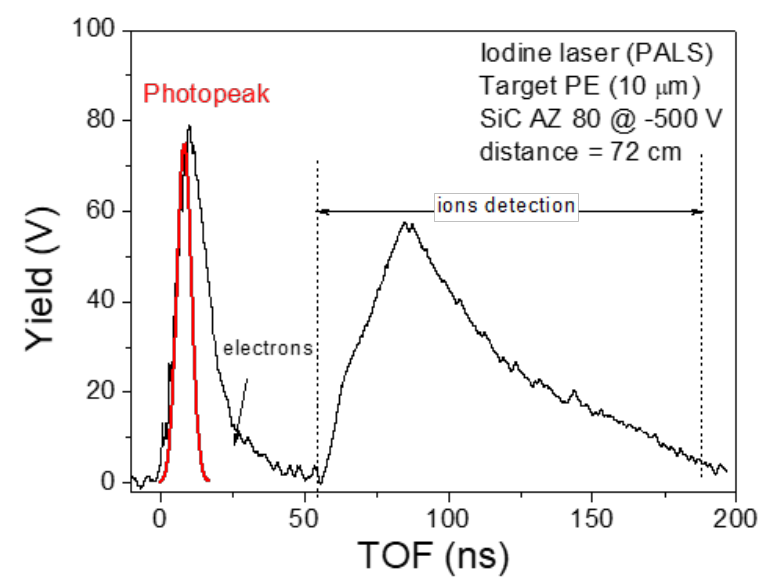

Fig. 2. TOF spectrum obtained at PALS facility irradiating PE target.

The two electron components can be distinguished using a Maxwell-Boltzmann distribution with two different temperatures:

$$
f(v)=\sqrt{\left(\frac{m_{e}}{2 \pi k_{B} T}\right)} 4 \pi v_{e}^{2} \exp \left[-\frac{m_{e} v_{e}^{2}}{2 k_{B} T}\right]
$$

where $m$ and $v_{e}$ are the electron mass and velocity, respectively.

Considering the ion acceleration due to charge separation effects in the plasma, the ions follow a Coulomb Boltzmann Shifted functions [7]:

$$
f(v)=A \sqrt{\left(\frac{m}{2 \pi k_{B} T}\right)^{3}} v^{3} \exp \left[-\frac{m}{2 k_{B} T}\left(v-v_{k}-v_{c}\right)^{2}\right]
$$

where $v$ represents the total velocity along the normal to the target surface and is due to the sum of thermal velocity $v_{t h}$, adiabatic expansion velocity $v_{k}$ and Coulomb velocity $v_{c}$.

\subsection{Target parameters}

The first analysis concerns the dependence of X-rays emission on the target composition. In the graphs of Fig. 3 are plotted the maximum yield as a function of the atomic number.

The spectra regard measurements performed both at low intensity (Nd:YAG laser in Messina) and at high intensity (Ti Sapphire laser at CELIA), were acquired using SiC IAZ 4 and SiC AZ 80, both in backward direction. In both situations a similar behavior was obtained and the experimental results follow the empirical law:

$$
\begin{gathered}
Y=15.47 \times[1-\exp (-0.0245 \times Z)] \\
Y=3.40 \times[1-\exp (-0.02 \times Z)]
\end{gathered}
$$

These results can be explained taking into account that increasing the atomic number of the target more electrons are injected into the plasma as a consequence of the ponderomotive force. The higher electron density promotes the particles acceleration with a consequent
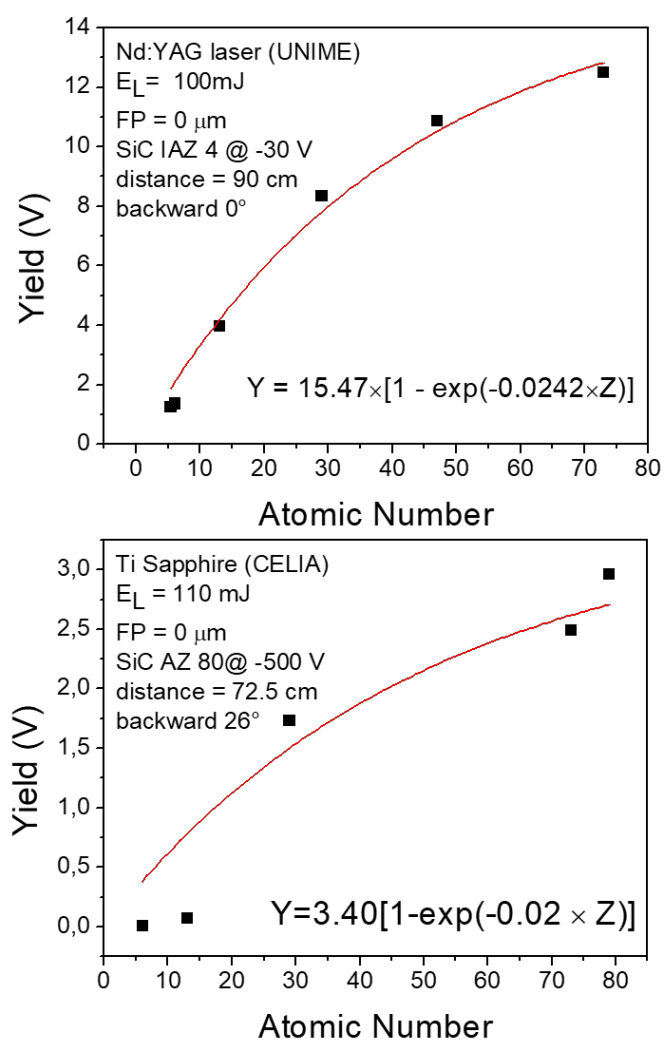

Fig. 3. Dependence of $X$-rays emission on the atomic number of the target.

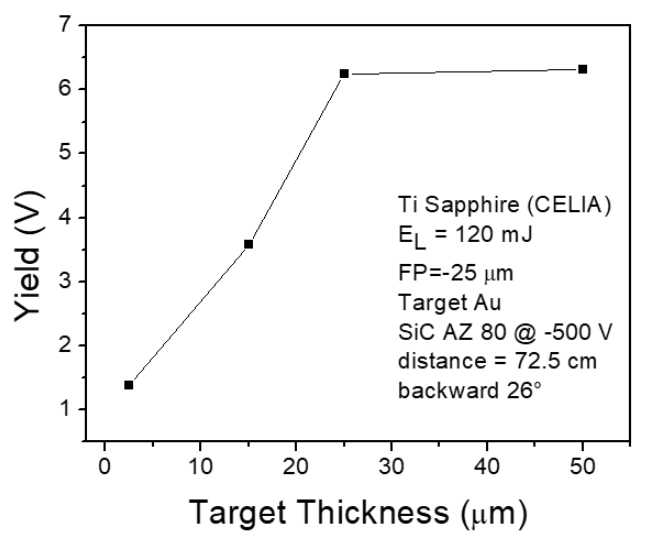

Fig. 4. Dependence of X-rays emission on the thickness of the target.

increase of the photon emission and of the plasma temperature.

Also the influence of the target thickness was evaluated and the experimental results were reported in Fig. 4.

In particular these data were obtained at high intensity laser, detecting the photons emitted in backward direction from Au targets $(2.48,15,25$ and $50 \mu \mathrm{m}$ thick). The graph shows an initial increase and then saturation.

In fact, increasing the thickness of the target the amount of material involved in the laser ablation processes also increases until, for thickness of about $30 \mu \mathrm{m}$, the saturation is reached. The saturation occurs when the target thickness is higher than the penetration depth of the laser into the target. 


\subsection{Laser parameters}

Many plasma parameters depend on the $I \lambda^{2}$ factor, being $I$ the laser intensity and $\lambda$ the laser wavelength [8]. In order to test the influence also on the X-rays emissivity, the laser intensity has been modified changing separately the laser energy, the focal position and the pulse duration, as reported in Fig. 5, Fig. 6 and Fig. 7, respectively. The spectra were obtained irradiating, at low laser intensity, a multilayer target consisting in Carbon and Nickel, while at high laser intensity a thin target of Gold was used. In both cases the detectors were placed in backward direction. As expected by increasing the laser power, a linear increase in photons yield is also observed.
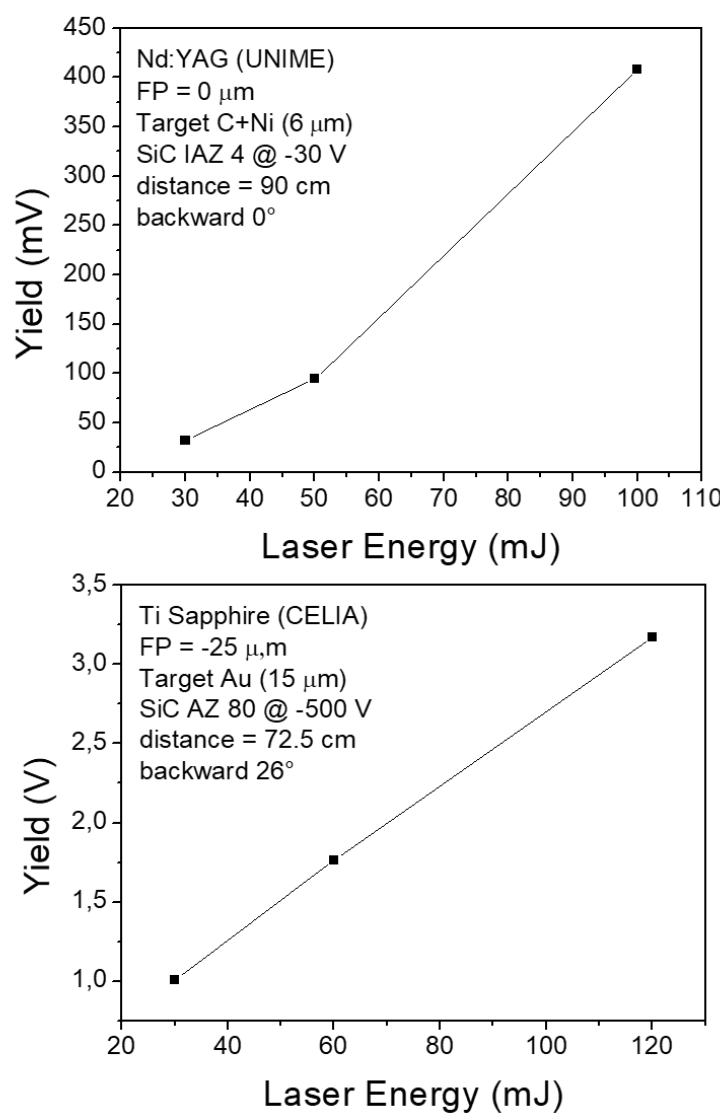

Fig. 5. Dependence of X-rays emission on the laser energy.

In fact, increasing the laser intensity, electrons can gain more energy leading to a greater emission of photons. Also the focal position plays an important role as can be seen in Fig. 6: the position in which laser is focused, with respect the target surface, not only determines the size of the laser spot but a proper choice of this distance can induce self-focusing effects and non-linear acceleration enhancement.

In this way it is possible to explain the presence of a second peak for $\mathrm{FP}=50 \mu \mathrm{m}$, in addition to the maximum obtained for $\mathrm{FP}=0 \mu \mathrm{m}$.

A similar dependence on the focal position was observed also for the ion yield as reported in literature [9].

Finally also the effects of pulse duration have been analyzed.

For low intensity laser, graph placed in the top of Fig. 7, were taken in consideration data from literature [10], in which the conversion efficiency was investigated as a function of the laser pulse duration, using a Nd:YAG laser

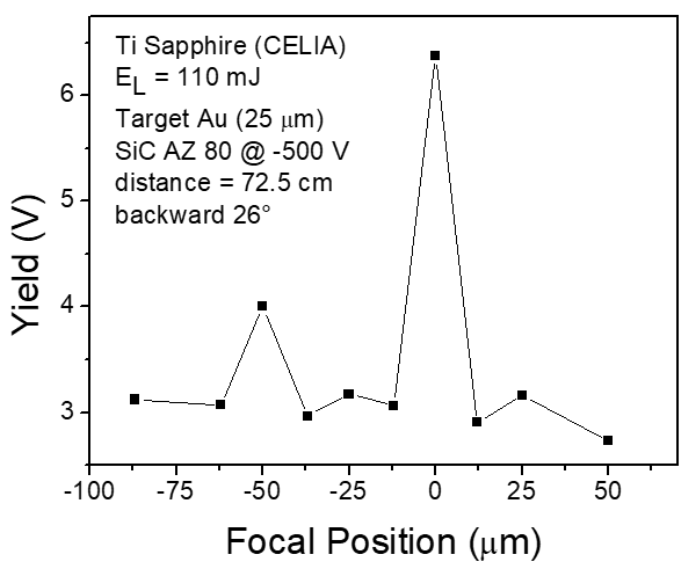

Fig. 6. Dependence of X-rays emission on focal position of the laser.
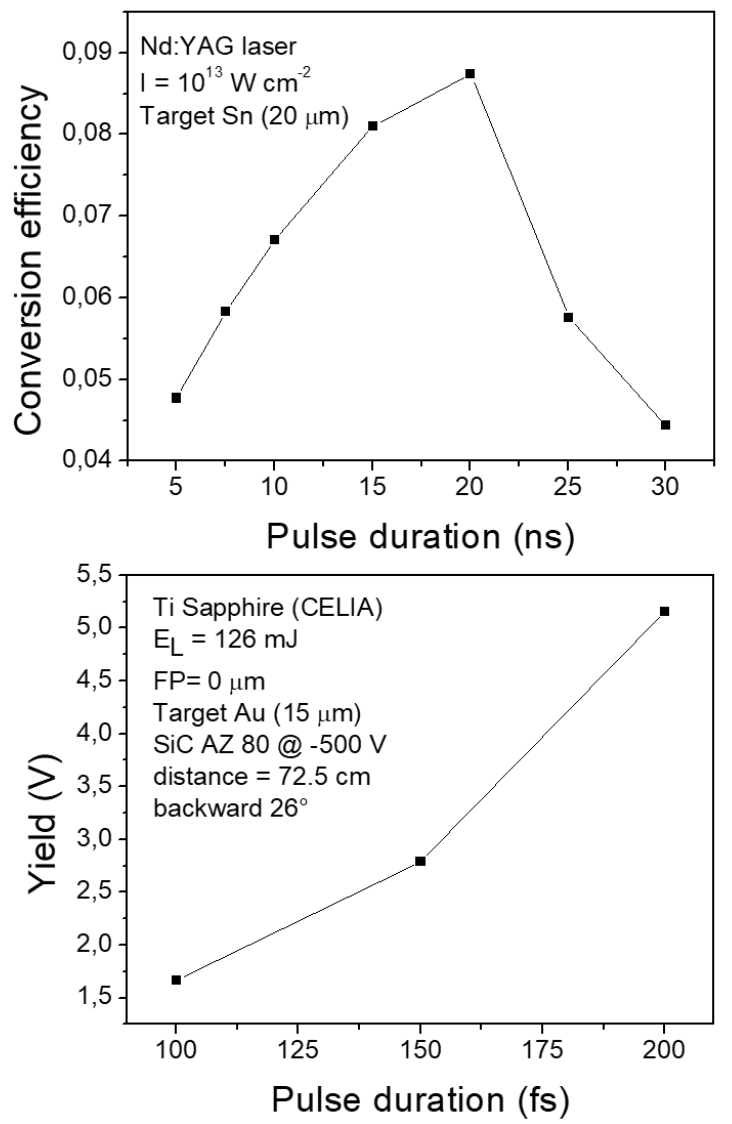

Fig. 7. Dependence of X-rays emission on the laser pulse duration.

with an intensity of $10^{13} \mathrm{Wcm}^{-2}$, focalized on a bulk Sn target. The photon emission increases with the pulse duration, because electron can interact for longer times with the laser pulse, gaining more energy. However, when the laser pulse becomes too long, the overall laser intensity decreases leading to the reduction of photon emission. For high intensity laser only the first region was investigated due to the constrains imposed by the laser configuration parameters. The lower and the higher pulse duration cannot be investigated with our method of analysis. 


\section{Conclusion}

In this work the X-rays emission of laser generated plasmas was characterized, analyzing how it is influenced by the target composition and laser parameters. Concerning the target influence, our data performed on Xrays can be combined with other works [11]-[13] about ions. Also in this case the same behavior was obtained: higher is the atomic number and higher is the total yield. Moreover, the analysis of experimental data shows also an increase of plasma temperature and of equivalent accelerating voltage for ions [14], confirming the considerations done about the increase of the electron current injected into plasma.

Data concerning the laser influence can be interpreted considering the $I \lambda^{2}$ factor. For example the ponderomotive energy that for high laser intensities represents the energy transferred to electrons is given by [8]:

$$
\varepsilon_{p}=\frac{e^{2} E_{0}^{2} \lambda^{2}}{16 \pi^{2} m_{e} c^{2}}=9.33 \times 10^{-14}\left(I \lambda^{2}\right) \text { in }(\mathrm{eV})
$$

where $e$ is the electron charge, $E_{0}$ the electric field of the electromagnetic wave, $m_{e}$ the electron mass and $c$ the speed of light in vacuum.

Many other plasma parameters depend on the $I \lambda^{2}$ factor, such as the shockwave pressure, the ion temperature, the emitted ion current and the electric field generated as a result of charge separation effects or the hot electron temperature, $k T_{\text {hot }}$, given by the relation [8]:

$$
k T_{\text {hot }}=\left(\sqrt{1+\frac{I \lambda^{2}}{2.8 \times 10^{18} W \mu^{2} / \mathrm{cm}^{2}}}-1\right) \cdot 511(\mathrm{keV})
$$

All the presented results show that the target parameters as well as the laser parameters play a fundamental role in the generation of X-ray from laser-target interaction.

This work was supported by the "Research and Mobility" project of Messina University No. 74893496, scientifically coordinated by Professor L. Torrisi.

\section{References}

1. D. Giulietti and L. A. Gizzi, La rivista del Nuovo Cimento, 21, (1998).

2. H.R. Griem, Plasma Spectroscopy (McGraw-Hill, New York, 1964).

3. D. Margarone, L. Torrisi, S. Cavallaro, E. Milani, G. Verona-Rinati, M. Marinelli, C. Tuvè, L. Làska, J Kràsa, M. Pfeifer et al., ECA F 31, (2007) P-5.011 .

4. M. Mazzillo, G. Condorelli, M. E. Castagna, G. Catania, A. Sciuto, F. Roccaforte, V. Raineri, IEEE Photonics Technology Letters, 21 23, (2009) 17821784.

5. A. Sciuto, L. Torrisi, A. Cannavò, G. Ceccio P. Musumeci, M. Mazzillo, L. Calcagno, Vacuum 131, (2016) 170-179.

6. A. Cannavò, L. Torrisi, JINST 11, (2016) C09005.

7. L. Torrisi, Radiations \& Defects in Solids, 171 1-2; (2016) 34-44.

8. L. Torrisi, Nukleonika, 60 (2), (2015) 207-212.

9. L. Làska, S. Cavallaro, K. Jungwirth, J. Kràsa, E. Krousky, D. Margarone, A. Mezzasalma, M. Pfeifer, K. Rohlena, L. Ryc, et al., Eur. Phys. J. D 54, (2009) 487-492.

10. B. Atalay, N. Kenar, A. Demir, Turk J Phys 33, (2009) 363-368.

11. J. Badziak, P. Parys, J. Wolowski, H. Hora, J. Kràsa, L. Làska, K. Rohlena, Optica Applicata, XXXV,(2005) 1.

12. G. Ceccio, JINST 11, (2016) C04017.

13. L. Torrisi, F. Caridi, L. Giuffrida, Laser and Particles Beams, 29, (2011) 29-37.

14. P. Musumeci, M. Cutroneo, L. Torrisi, A. Velyhan, M. Zimbone, L. Calcagno, Phys. Scr. T 161 (2014) 014021. 